\title{
Re: Unprepared, Understaffed, and Unplanned
}

\author{
Edward P. Taubman
}

Received: 27 December 2009/Accepted: 24 February 2010/Published online: 1 April 2010

(C) National Society of Genetic Counselors, Inc. 2010

I appreciate the issues that Robert Resta has importantly raised, Wendy Uhlmann's comments, and Heather Hampel's sharing her experiences. I certainly agree that, when the time comes, an improved team effort of patients and counselors will be necessary. However, we haven't said much about the two major players who really need to be on the team: the testing companies and the insurers. They both already have the information infrastructure to identify patients, the personnel to track them down, and the funds to send the message out over the print, radio, and TV media. I believe that companies that are presently charging thousands of dollars to perform, and report, these mostly uninformative tests, have a responsibility to use their resources to help when more informative testing becomes available. Furthermore, the insurance companies who, in the name of best patient care, have created policies and infrastructure to control who is presently eligible for these tests, and have required information about family histories to limit access to these tests, also have a responsibility to use those same resources to help inform their clients when something more informative comes along.

The issue of storing DNA samples for future use by a patient's relatives is potentially important and increasingly being raised. For those of us not involved with day to day research, the logistics of this is limiting how often it is done. Again, it would be much easier for all of us if that process were facilitated by the companies that already have the infrastructure to collect, store, and track DNA samples. Compared to the present system, think about how much simpler and efficient it would be if on the BRCA or HNPCC ordering sheet there were a check mark to store the patient's DNA. Such a process would be voluntary, a reasonable fee could be charged to those patients who chose to participate, and we would all have a centralized place to look should the need to test a sample arise in the future.

E. P. Taubman $(\bowtie)$

General Internal Medicine \& Cancer Genetic Counseling,

Olney Medical Associates, L.L.C.,

18109 Prince Philip Drive, Suite 275,

Olney, MD 20832, USA

e-mail: cancergenedoc@olneymedical.com 